\title{
Towards better base editing
}

\author{
The editing of single DNA bases in the genome is being optimized for higher editing precision and versatility.
}

$\Lambda$ $s$ the first CRISPR-mediated gene editing therapies enter clinical trials, optimizing them for safety, efficiency and better capabilities becomes paramount. New variants of CRISPR-associated (Cas) nucleases, such as xCas9 and SpCas9-NG, developed to target alternative protospacer adjacent motif (PAM) sequences, broaden the range of genomic loci that can be edited. Base editors - which modify base pairs at specific sites by using a catalytically dead Cas nuclease fused to a nucleobase deaminase enzyme, as well as a guide RNA to target specific DNA sequences - typically do not generate doublestrand breaks (which nucleases such as Cas9 do) and thus work independently of the errorprone cellular DNA machinery. Cytosine base editors (CBEs), which convert $\mathrm{C} \bullet \mathrm{G}$ pairs to T•A pairs, and adenine base editors (ABEs), which convert $\mathrm{A} \bullet \mathrm{T}$ pairs to $\mathrm{G} \bullet \mathrm{C}$ pairs, do not require a DNA template to operate and are more efficient at editing the DNA of quiescent cells than standard Cas nucleases, which require endogenous DNA repair processes (such as homology-directed repair via a DNA template) that are inefficient in non-dividing cells and prone to generating uncontrolled insertions and deletions (indels).

Despite these advantages over commonly used Cas9 nucleases, base editors share many of the limitations of other genomeediting agents - in particular, limitations associated with their in vivo delivery to target tissues. Although adeno-associated viruses (AAVs) are among the most effective delivery vehicles for proteins and nucleic acids, the large size of base editors preclude their packaging into individual AAV capsids. In an Article published in this issue, David Liu and colleagues describe the in vivo delivery of ABEs and CBEs, each split into two portions and incorporated in a dual AAV system that overcomes the packaging limitations of AAV vectors. Upon reaching the target cells, the split ABEs or CBEs are reconstituted by trans-splicing inteins. This delivery strategy allowed the researchers to achieve efficient base editing in the brain, liver, retina, heart and skeletal muscle of mice, and, in particular, the correction of a mutation in the NPC1 gene (associated with the neurodegenerative disease NiemannPick type C1) in the mouse brain, which slowed disease progression and increased lifespan in the treated mice.

A second Article included in this issue, authored by Wen Xue, Hao Yin, David Liu

\begin{abstract}
GAGGTACACCAGCACCAAAGAGGTGCTG GTACGAGACACGGATCGACCTGTCTCAGI ACTAATCTGAGCGACATCAT AGTCCATCCTGATGCTGCCTGAGGAGGTC ¿CTGGTGCACACCGCCTACGACGAGTCC, CCCGAGTATAAGCCTTGGGCCCTGGTCA IGAGCGGAGGATCCAAAAGAACCGCCGAI TCTAAGATCTGATAATCAACCTCTGGATT, TGTTGCTCCTTTTACGCTATGTGGATACG! ATGGCTTTCATTTTCTCCTCCTTGTATAAA :GCCTGCCTTGCCCGCTGCTGGACAGGGC ¿TGTGCCTTCTAGTTGCCAGCCATCTGTTI 3GTGCCACTCCCACTGTCCTTTCCTAATA TCATTCTATTCTGGGGGGTGGGGTGGGG CAGGCATGCTGGGGATGCGGTGGGCTCT ЗGCCACTCCСTCTCTGCGCGCTCGCTCG(
\end{abstract}

Credit: Figure reproduced from Liu, D. et al. Nat. Biomed. Eng. https://doi.org/10.1038/s41551-0190501-5 (2020), Springer Nature Ltd

and co-workers, involves the use of a single guide RNA (sgRNA) and an ABE for the correction, in mice, of an A-to-G splice site mutation associated with the deficient production of fumarylacetoacetate hydrolase (FAH) and the development of tyrosinaemia (characterized by a high concentration of the amino acid tyrosine, a building block of most proteins, in the blood). The sgRNA and $\mathrm{ABE}$ were administered into adult mice hydrodynamically via the tail vein so as to target the liver (that is, by generating hydrodynamic pressure to cause the rapid injection of a large volume of fluid containing the sgRNA and ABE plasmids). Although the procedure corrected the Fah mutation in only a relatively small fraction of hepatocytes $(<10 \%)$, this was enough to maintain the body weight of the treated mice and keep them alive in the absence of a drug (an inhibitor of the tyrosine catabolic pathway upstream of FAH) that is otherwise required for survival. Such robust phenotypic rescue was most likely a result of the selective proliferation of the edited hepatocytes in the liver, despite the modest fraction of initially edited cells. The researchers then developed a chemically modified sgRNA and a codon-optimized $A B E$ that surpassed the editing efficiency of the original $\mathrm{ABE}$, and devised a lipidnanoparticle formulation that delivered these optimized components to the liver of mice. This suggests that lipid-based delivery systems, previously used for the delivery of other CRISPR systems, are also viable for in vivo base editing.

The applicability of base editors is constrained by the PAMs that determine where Cas nucleases can bind to DNA. Hence, base pairs that lack an appropriate PAM within their genomic region are out of reach for base editors. However, laboratoryevolved or engineered Cas variants (such as xCas9 or SpCas9-NG) possessing different PAM requirements can broaden the range of genomic sites that can be targeted. In a third Article published in this issue, Hyongbum Henry Kim and colleagues present the findings of a broad assessment of the PAM compatibility and of the on-target and off-target activities of SpCas9, xCas9 and SpCas9-NG in human cells. The researchers identified non-NGG PAM sequences that can be used by SpCas9-NG and SpCas9 to edit six sites associated with genetic diseases that had not been targetable by base editors. They also developed deep-learning models for the prediction of the activities of $\mathrm{xCas} 9$ and SpCas9-NG at target sequences.

The hard job of easing or removing performance and applicability constraints in CRISPR-based therapies, as exemplified by the articles highlighted above in the case of base editing, are crucial if CRISPR-based therapies are to be successfully applied in humans. Yet some constraints cannot be solved via rational tweaking or optimization strategies; instead, they require step-change innovations. Indeed, although base editing cannot make all of the eight 'transversion' base-to-base-conversions (such as T-to-A and A-to-C), targeted insertions or targeted deletions, this has been bypassed with prime editing (Nature 576, 149-157; 2019), a new editing technology recently developed by David Liu's team. Prime editing uses an engineered guide RNA that both specifies the target locus and encodes the desired edit, together with a deactivated Cas9 fused to a reverse transcriptase, to copy the desired edit into the target locus. Although performance-wise this more versatile search-and-replace genome editor lags the latest base editors in some respects (in particular, current base editors can be more efficient and lead to fewer indels than prime editors), it will surely be optimized by following in the footsteps of base editing.

Published online: 14 January 2020 https://doi.org/10.1038/s41551-020-0515-Z 